\title{
Insecticide resistance testing in malaria vectors in Tanzania: Challenges in mosquito sampling and rearing under field conditions
}

\author{
Basiliana Emidi ${ }^{1,2 *}$, Bilali Kabula ${ }^{4}$, Patrick Tungu ${ }^{3}$, Julius Massaga ${ }^{2}$, William Kisinza ${ }^{3}$ \\ ${ }^{1}$ Kilimanjaro Christian Medical College of Tumaini University, Moshi, Tan₹ania \\ ${ }^{2}$ National Institute for Medical Research, Headquarters, Dar es Salaam, Tanzania \\ ${ }^{3}$ National Institute for Medical Research, Amani Research Centre, Muheza, Tanzania \\ ${ }^{4}$ National Institute for Medical Research, Tukuyu Research Centre, Tukuyu, Tanzania
}

\begin{abstract}
Background: The National Institute for Medical Research, Amani centre, in collaboration with National Malaria Control Programme, has been conducting annual insecticide resistance surveillance since 1999, aimed at early detection of resistance to insecticides used for malaria control in Tanzania. The Standard WHO method for larvae collection and rearing were used but challenges and limitations were encountered. For example rearing the larvae and adult mosquitoes using the Standard WHO method experienced 100\% mortality for larvae; and adults in three days. The researchers therefore made modifications to the Standard WHO method to create suitable tools for the field environment. Methods: A ladle was created from an empty water bottle in which an oval hole longitudinally cut halfway from the bottom. Instead of using TetraMin as mosquito larval food, green algae were collected from mosquito breeding sites and used as larval food. Improvised heater of charcoal stoves and humidifier of wet fabric such as "Kanga" and "Kitenge" were also used. Results and conclusion: There was 90\% larval survival, adult mosquito survived much better and the scientists had a total of 467 mosquitoes to run the insecticide susceptibility tests. Innovative ways are necessary under field conditions for mosquito breeding in susceptibility studies.
\end{abstract}

Key words: Malaria, Anopheles gambiae complex, larvae, fabric, resistance, susceptibility, Tanzania.

\section{Introduction}

In spite of technological advances in diagnosis, prevention and treatment, malaria is still one of the biggest global killers. It is estimated that about 219 million cases and 660000 deaths of malaria occurred in 2010. Africa is the most affected continent with about $90 \%$ of all malaria deaths. Malaria is one of the major causes of morbidity and mortality in Africa (Casimiro, et al., 2004; WHO, 2012). In Tanzania, malaria remains one of the major public health problems. It is the main cause of admission for children (38\%) and adults (32\%) in health facilities (Gallup \& Sachs, 1998; Magesa et al., 2005; Rugemalila et al., 2006) Vector control is a major component of the global strategy for malaria control which aims to prevent parasite transmission mainly through interventions targeting adult Anopheline vectors (RBM, 2010). Currently, the National Malaria Control Programme of Tanzania is promoting large scale use of insecticide-treated nets as the main vector control tool. Ongoing strategies rely heavily on the use of safe and effective insecticides through indoor residual spraying or insecticide-treated nets. The successful implementation of these strategies requires good knowledge of vectors distributions, biology, and susceptibility to available insecticide compounds.

In many African countries, Anopheline mosquitoes are developing resistance to all classes of insecticides used for mosquito control (Hunt et al., 2011; Kabula et al., 2012). Among these, Pyrethroids are the only option for net treatment due to their relative safety for humans at low dosage, excito-repellent properties, rapid rate of knock-down, and killing effects (Zaim et al., 2000). The emergence and rapid spread of Pyrethroid resistance in An. gambiae complex populations may be a threat for the sustainability of malaria vector control activities (Hunt et al., 2011; Temu et al., 2012). This raises the need for national surveys for monitoring the insecticide susceptibility status of major vectors, detection of resistance possible genes and assessing their implications on vector control activities (Kelly-Hope \& Ranson, 2008). The National Institute for Medical Research, Amani Research Centre in collaboration with National Malaria Control Programme and other higher learning and research institutions has been conducting annual insecticide resistance surveillance since 1999 with the aim of early detection and containment of resistance

*Corresponding author: bemidi@nimr.or.tz 
to insecticides used for malaria control in Tanzania. In 2012, the Bariadi district was included as one of the sentinel sites for this important activity. In monitoring of insecticide resistance to malaria vectors, the susceptibility tests needed to be conducted. To achieve such an important goal, the institute has been implementing the activities in selected sentinel sites around mainland Tanzania. Screening for insecticide susceptibility is the initial step towards designing proper resistance management in any setting. This can only be achieved by undertaking a systematic resistance surveillance which by itself has aims to provide baseline data for program planning and insecticide selection before the start of control interventions.

In using the Standard WHO method for the sampling and rearing of mosquitoes (WHO, 1975), some challenges and limitations were encountered during the field work conducted in Bariadi District from July to August, 2012. First, it was found that most of the Anopheles gambiae complex breeding sites were about to dry, with only small amounts of water remaining on rocks and in livestock hoof prints. Secondly, there were massive deaths of reared Anopheles gambiae complex larvae and adult. It was observed that larval mortality was quite high during the process of rearing before reaching day 3 . The mortality was even higher after provision of TetraMin (fish food) and some larvae tended to bite each other regardless of their developmental stages even after separating the different instars. Starvation and injuries were suspected to be contributing to the observed mortality among the larvae. The emerged adult mosquitoes were dying in large numbers before reaching the age of 3 days which is the recommended age range for conducting susceptibility testing. Disappointingly, $100 \%$ mortality continued unabated within 12 hours of emergence despite the efforts to change the cages and even replace the netting with a new wedding mesh fabric just in case there had been insecticidal contamination.

In order to overcome these obstacles, some improvisions were introduced and modifications were made to the current WHO standard methods of collecting and rearing mosquitoes. The current paper reports the alternative techniques used in order to improve the outcome.

\section{Methods}

\section{Study area}

The study was conducted in the Bariadi district between July and August, 2012. Bariadi district is situated at $2.8^{\circ}$ South latitude, $33.98^{\circ}$ East longitude and 1,272 meters above sea level. It is a small town in the Simiyu region in Tanzania (Figure 1). The Bariadi community depends on livestock keeping and crop farming to earn their living.

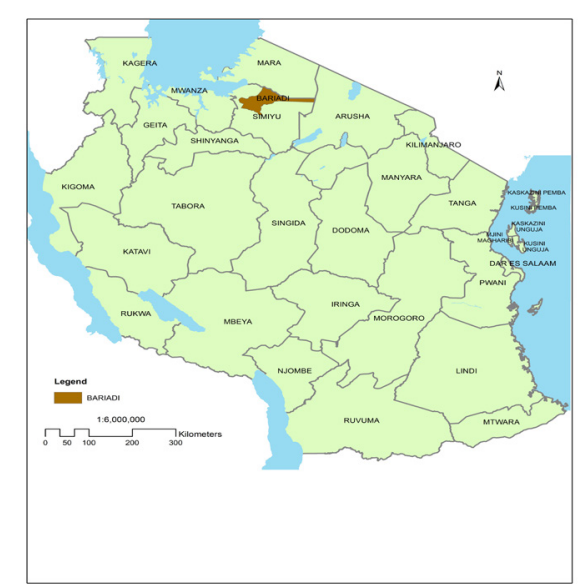

Figure 1. Map of Tanzania showing the Bariadi district

\section{Study sites}

Mosquito larvae were collected from three types of breeding sites namely river banks, hoof prints and on rocks.

\section{Mosquito larval collection and rearing}

Rearing of mosquitoes for the susceptibility tests was achieved by using improvised tools and methods. A WHO Standard Dipper, was replaced by a ladle made from an empty $350 \mathrm{ml}$ sized water bottle by longitudinally cutting an opening to make an oval hole halfway long from the bottom. Sometimes for larval collection, table ladles were also used depending on the breeding site depth (Figure 2, 3 \& 4). TetraMin mosquito larval food, was replaced by green algae collected from mosquito breeding sites. The mosquito larvae were sorted into separate containers according to instars to reduce possible predation by using a pipette. Larvae were kept in white bowls until pupa had developed. Pupa were collected by using a pipette and placed in containers with some water and transferred to the prepared cages until adult mosquitoes had emerged. 


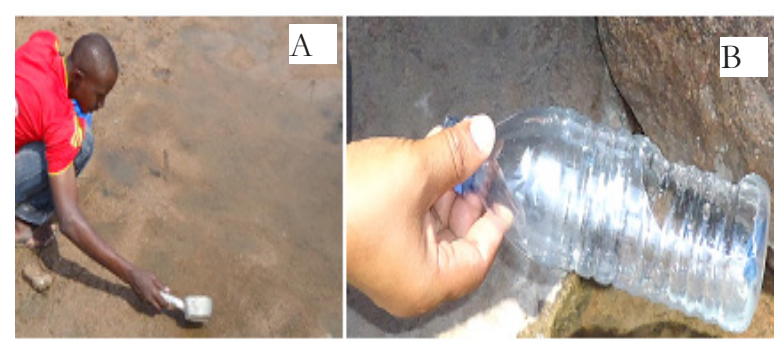

Figure 2. The use of an improvised dipper a standard dipper and B-Improvised empty $350 \mathrm{ml}$ sized plastic bottle of water dipper

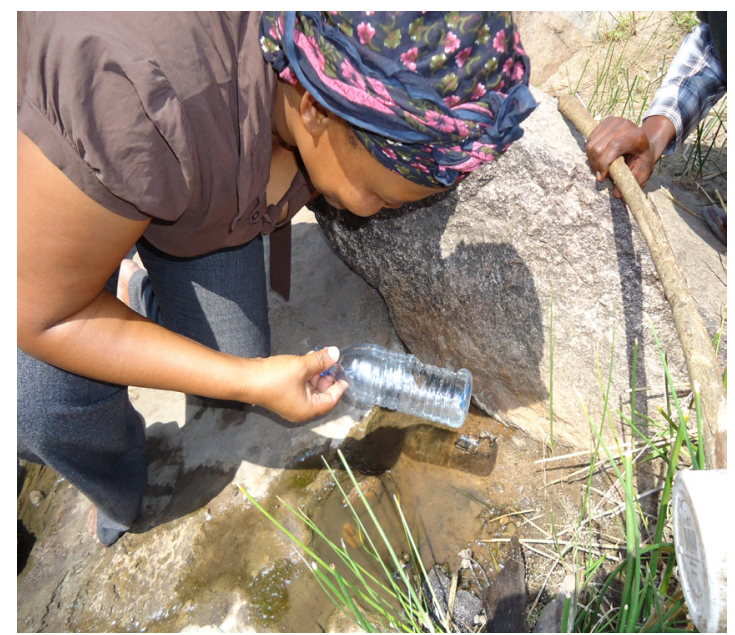

Figure 3. Larvae collection tools - an improvised empty plastic bottle

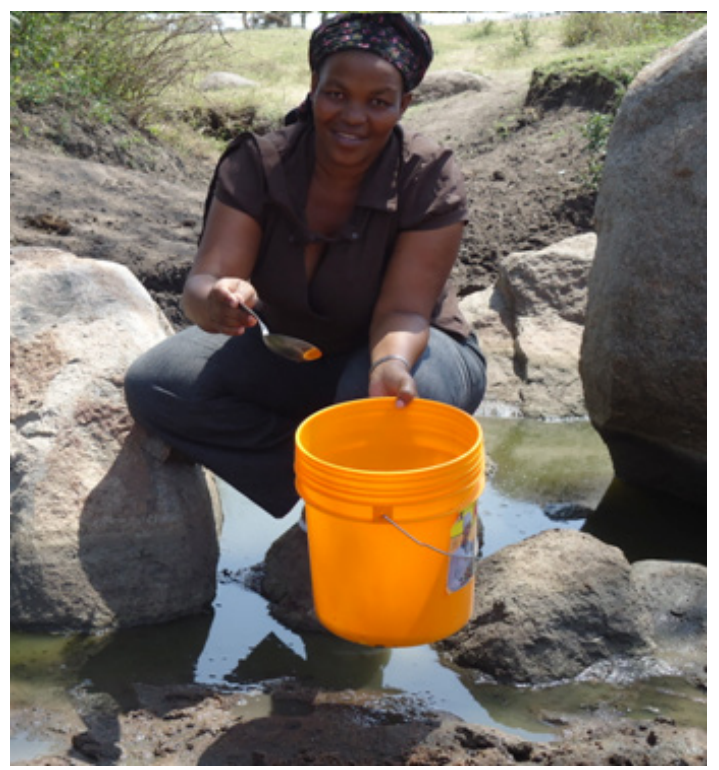

Figure 4. Larvae collection tool - a table ladle

In Bariadi district, in July and August,2012 the weather was cool and dry; hence we thought that optimal temperature and humidity for development of mosquitoes could not be attained without further input.
Therefore, we used improvised room heaters of charcoal stoves and improvised humidifiers of wet cotton fabric namely "kanga" and "vitenge" to cover the cages, and kept continuously boiling water in the room using aluminium pots to maintain high humidity in the room for adult mosquitoes to survive well (Figure 5).

The emerged adult mosquitoes were fed on glucose solution in cotton wool prepared as per standard operating procedures (WHO, 1975). Because this study was conducted in the field where there was no insectary, an effort was made to prevent the emerged adult mosquitoes from being eaten by ants. For that purpose, ant control was achieved by using a basin full of water with a bucket in it (Figure 6). This bucket was filled with water too and covered to prevent it from floating. Sometimes we used a four-legged stool to hold a basin full of water as well. Then a mosquito cage was placed on the bucket with or without supporting bars depending on the size of the cage.

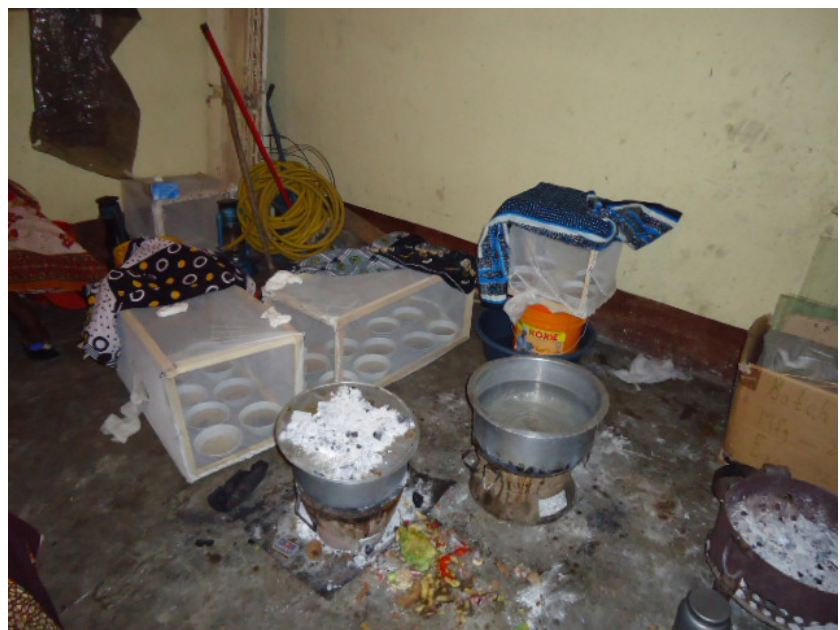

Figure 5. A - Mosquito cage preparation, B - Improvised insectary, room heater (charcoal stove), humidifier of wet Kanga and Vitenge, continuous boiling water to maintain humid air.

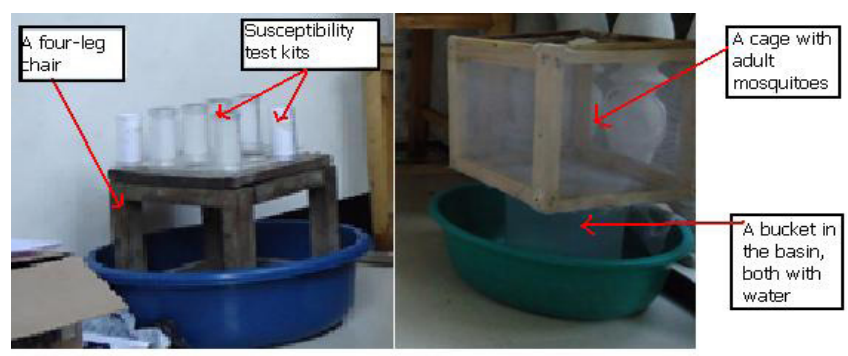

Figure 6. Mechanisms of ant control to prevent eating of mosquitoes 


\section{Results}

The improvised dippers were efficient enough to collect water with larvae from even the quite small water bodies on rocks and in hoof-prints. The use of the WHO standard dipper was not suitable for collection of Anopheles gambiae complex larvae in such shallow breeding sites with small amount of water as that found on the rocks. Algae provided a good source of nutrients for the larvae of all instars, and we recorded an encouraging $90 \%$ survival of larvae and adequate number of adult emergences from the pupa. The humidity was kept high enough to maintain the colony of mosquitoes alive for more than three days until they were ready for susceptibility testing. As a result, a total of 467 Anopheles gambiae complex adults survived and were used for that purpose. The basin full of water served well as an anti- ant trap as no ants crossed the water barrier into the mosquito cages or into the tubes holding mosquitoes for susceptibility tests over the 24 hour holding period for the test.

Five types of insecticides were tested for susceptibility to female adult Anopheles gambiae complex mosquitoes. These insecticides were lamdacyhalothrine $0.05 \%$ (pyrethroid), permethrine $0.75 \%$ (pyrethroid), DDT 4\% (organochlorine), fenitrothion 1.0\% (organophosphate) and bendiocarb $0.1 \%$ (carbamate). All the procedures for conducting the susceptibility test were according to the WHO 1998 Standard Operating Procedures (WHO, 1998). The numbers of Anopheles gambiae complex mosquitoes exposed to each insecticide are indicated in Table 1 below.

Total 1. Number of Anopheles gambiae complex testes per insecticide

\begin{tabular}{|c|c|c|c|c|c|c|}
\hline No. mosquitoes & $\begin{array}{l}\text { Lamdacy- } \\
\text { halothrine } 0.05 \%\end{array}$ & $\begin{array}{l}\text { Permethrine } \\
0.75 \%\end{array}$ & $\begin{array}{l}\text { DDT } \\
4 \%\end{array}$ & $\begin{array}{l}\text { Fenitrothion } \\
1.0 \%\end{array}$ & $\begin{array}{l}\text { Bendiocarb } \\
0.1 \%\end{array}$ & Total \\
\hline $\begin{array}{l}\text { Killed at the end of the holding } \\
\text { period (after } 24 \text { hours) }\end{array}$ & 67 & 95 & 94 & 100 & 98 & 454 \\
\hline Survived after 24 hours & 7 & 3 & 3 & 0 & 0 & 13 \\
\hline Total exposed & 74 & 98 & 97 & 100 & 98 & 467 \\
\hline
\end{tabular}

\section{Discussion}

Human activities such as animal keeping and climatic conditions have a great influence on mosquito breeding. During the dry season, most water bodies dry up and only a few mosquito larvae can be found in small and tiny water collections (Mala \& Irungu, 2011; Mala et al., 2011). In the wild, mosquito larvae develop and thrive in a large variety of habitats. A major source of nutrients for mosquito larvae is plant material that has been already degraded by fungi or bacteria (Benedict, 2007; Sattler et al., 2005). However, there were breeding sites which were a little larger such as sunlit stagnant waters in river banks. Such sites as found in river banks are known to contain much green algae, some mosquito larvae, and mosquito larval predators as well (Sattler et al., 2005). The presence of green algae in the breeding site can be an indicator of the presence of the mosquito larvae (Bugoro et al., 2011; Mala et al., 2011). This was also the case during implementation of our study, whereby the presence of Anopheles gambiae complex larvae was associated with the presence of green algae. It is for this reason that we opted to use green algae as source of food for the collected larvae. These findings are supported by previous study in western Kenya showing that water nutrient and algal content in larval habitats of Anopheles gambiae complex play important roles in their resource ecology (Mala \& Irungu, 2011). However, for Anopheles gambiae complex larval growth and adult survival, there are optimal temperature, humidity and light conditions required (Bugoro et al., 2011; Koenraadt et al., 2003). In the presence of an insectary, it is easier to control these optimum conditions than in the rural field environment (Dida et al., 2015).

\section{Conclusion}

Standard WHO methods for sampling and rearing mosquitoes may not be suitable in all field settings for various purposes such as monitoring of insecticide resistance for malaria vectors. It can be used as a guide and may need to be modified according to the setting and season. Based on these findings, it is recommended that scientists be more innovative by using locally available resources to develop techniques which could suitably address the field challenges encountered and not addressed by the present WHO guidelines. The challenges and results highlighted in this paper also call for the periodic review of the WHO guidelines to incorporate innovative experiences from the field. 


\section{Acknowledgements}

The authors wish to acknowledge the invaluable contributions and support from various people and institutions. We also express our gratitude to the District Malaria Focal Person, the District Executive Director (DED) and the District Medical Officer (DMO) in the Bariadi District for their support. The work described here was supported by the U.S. President's Malaria Initiative through the U.S. Agency for International Development under the RTI International Tanzania Vector Control Scale-Up Project $(621-\mathrm{A}-00-10-00015-00)$.

\section{Authors' contributions}

Both authors worked on literature review and hereafter, $\mathrm{BE}$ set the first draft of this manuscript and received substantial comments from WK, JM, BK and PT.

\section{Declaration}

The authors declare no conflict of interest in relation to the presentation of this manuscript.

\section{References}

Benedict M. (2007). Methods in Anopheles Research. Atlanta: CDC. Bugoro, H, Hii, J, Russell, TL, Cooper, RD, Chan, BKK, Iro'ofa, C, Butafa, C, Apairamo, A., \& Bobogare, A \& Chen, C. (2011). Influence of environmental factors on the abundance of Anopheles farauti larvae in large brackish water streams in Northern Guadalcanal, Solomon Islands. Malaria Journal, 10(1), 262. doi:10.1186/1475-2875-10-262

Casimiro, S. L. R., Hemingway, J., Sharp, B. L., \& Coleman, M. (2007). Monitoring the operational impact of insecticide usage for malaria control on Anopheles funestus from Mozambique. Malaria Journal, 6, 142. doi:10.1186/14752875-6-142

Dida, G. O., Gelder, F. B., Anyona, D. N., Abuom, P. O., Onyuka, J. O., Matano, A.-S., Ofulla, A. V. (2015). Presence and distribution of mosquito larvae predators and factors influencing their abundance along the Mara River, Kenya and Tanzania. SpringerPlus, 4(1). doi:10.1186/s40064-0150905-y

Gallup, J. L., \& Sachs, J. D. (1998). The Economic Burden of Malaria : Cross-Country Evidence, 1994(1997), 1-21.

Hay, SI, Guerra, CA, Tatem, A., \& Noor, AM \& Snow, R. (2004). The global distribution and population at risk of malaria : past, present, and future. Lancet, 4, 327-336.

Hunt, R. H., Fuseini, G., Knowles, S., Stiles-Ocran, J., Verster, R., Kaiser, M. L., Coetzee, M. (2011). Insecticide resistance in malaria vector mosquitoes at four localities in Ghana, West Africa. Parasites \& Vectors, 4(1), 107. doi:10.1186/17563305-4-107
Kabula, B., Tungu, P., Matowo, J., Kitau, J., Mweya, C., Emidi, B., Kisinza, W. (2012). Susceptibility status of malaria vectors to insecticides commonly used for malaria control in Tanzania, 17(6), 742-750. doi:10.1111/j.1365-3156.2012.02986.x

Kelly-Hope, L., Ranson, H. \&, \& J., H. (2008). Lessons from the past: Managing Insecticide resistance in malaria control and eradication programmes. Lancet Infect Dis., 8, 387-389.

Koenraadt, CJM, Paaijmans, KP, Githeko, AK, Knols, G. \&, \& Takken, W. (2003). Egg hatching, larval movement and larval survival of the malaria vector Anopheles gambiae in desiccating habitats. Malaria Journal, 2(20), 1-9.

Magesa, S. M., Lengeler, C., deSavigny, D., Miller, J. E., Njau, R. J. a, Kramer, K., Mwita, A. (2005). Creating an "enabling environment" for taking insecticide treated nets to national scale: the Tanzanian experience. Malaria Journal, 4, 34. doi:10.1186/1475-2875-4-34

Mala, A. \&, \& Irungu, L. (2011). Factors influencing differential larval habitat productivity of Anopheles gambiae complex mosquitoes in a western Kenyan village. Journal of Vector Borne Diseases, 48(1), 52-57.

Mala, A. O., Irungu, L. W., Shililu, J. I., Muturi, E. J., Mbogo, C. C., Njagi, J. K., \& Githure, J. I. (2011). Dry season ecology of Anopheles gambiae complex mosquitoes at larval habitats in two traditionally semi-arid villages in Baringo, Kenya. Parasites \& Vectors, 4(1), 25. doi:10.1186/1756-3305-4-25

RBM. (2010). Scaling-up insecticide-treated netting programmes in Africa. Roll Back Malaria, (April 2000).

Rugemalila, J. B., Wanga, C. L., \& Kilama, W. L. (2006). Sixth Africa Malaria Day in 2006: how far have we come after the Abuja Declaration? Malaria Journal, 5, 102. doi:10.1186/14752875-5-102

Sattler, M. A., Mtasiwa, D., Kiama, M., Premji, Z., Tanner, M., Killeen, G. F., \& Lengeler, C. (2005). Habitat characterization and spatial distribution of Anopheles sp. mosquito larvae in Dar es Salaam (Tanzania) during an extended dry period. Malaria Journal, 4, 4.

Temu, E. a., Maxwell, C., Munyekenye, G., Howard, A. F. V, Munga, S., Avicor, S. W., Ranson, H. (2012). Pyrethroid Resistance in Anopheles gambiae, in Bomi County, Liberia, Compromises Malaria Vector Control. PLoS ONE, 7(9), 5-10. doi:10.1371/journal.pone.0044986

WHO. (1975). Manual on Practical Entomology in Malaria.

WHO. (1998). Testing procedures for Insecticide Resistance Monitoring in Malaria Vectors, Bio- Efficacy and Persistence of Insecticides on Treated Surface. Geneva.

WHO. (2012). World Malaria Report 2012 Fact Sheet Geneva.

Zaim, M., Aitio, A. \& Nakashima, N. (2000). Safety of Pyrethroid-treated mosquito nets. Med Vet Entomol, 14, 1-5. 